\title{
CARACTERIZAÇÃO MECÂNICA E METALÚRGICA DAS LIGAS TIXOCONFORMADAS AA7004 E AA7075
}

Luis Vanderlei Torres', 2

Eugênio José Zoqui ${ }^{2}$

\section{Resumo}

A crescente necessidade da redução de custos nas indústrias, como também a melhoria da qualidade dos produtos finais têm convergido para o surgimento de técnicas que visam o aperfeiçoamento ou mesmo a substituição dos processos convencionais de fabricação existentes, reduzindo as etapas envolvidas na obtenção dos produtos e consequentemente reduzindo os custos envolvidos. Por esse motivo, o processo de tixoconformação, ou seja, o processamento de ligas metálicas no estado de coexistência de fase sólida e fase líquida têm ganhado importância nos últimos anos. Este trabalho tem como objetivo analisar o comportamento mecânico e metalúrgico através de ensaios de dureza vickers das ligas tixoconformadas AA7004 e AA7075. As ligas tixoconformadas quando comparadas com a condição fundida (como recebida) apresentaram valores elevados de dureza, fato atribuído à compactação do material. Com a realização do tratamento térmico de solubilização e envelhecimento há novamente o aumento no valor da dureza, chegando a valores em torno de $140 \mathrm{HV}$ para a liga AA7004 e em torno de $160 \mathrm{HV}$ para a liga AA7075, tornando-se matérias-primas promissoras para o processo de tixoconformação.

Palavras-chave: Tixoconformação; Solubilização e envelhecimento; AA7004; AA7075.

\section{MECHANICAL AND METALLURGICAL CHARACTERIZATION OF AA7004 AND AA7075 THIXOFORMING ALLOYS}

\begin{abstract}
The increasing need for cost reduction in the industries, as well as the improvement of the quality of the final products, have converged to the arising of techniques that aim the improving or even replacing the existing conventional manufacturing processes, reducing the steps involved in obtaining the products and consequently reducing the involved costs. For this reason, the thixoforming process, that is, the processing of metallic alloys in the coexistence state of solid and liquid phase have gained importance in last years. This work aims to analyze the mechanical and metallurgical behavior through vickers hardness tests of AA7004 and AA7075 thixoforming alloys. The thixoforming alloys when compared to the casting condition (as received) presented elevated values of hardness, wich is an attributed fact to the material compaction. With the heat treatment of solubilization and aging there is again the increase in the hardness value, reaching values around I $40 \mathrm{HV}$ to the AA7004 alloy and around I60 HV to the AA7075 alloy, becoming both alloys promising raw materials to the thixoforming process.
\end{abstract}

Keywords: Thixoforming; Solubilization and aging; AA7004; AA7075.

\section{INTRODUÇÃO}

O alumínio é o metal não ferroso mais produzido no mundo em volume e o segundo metal mais utilizado pela indústria, perdendo apenas para o aço; sua utilização está ligada às excelentes propriedades apresentadas, tais como, resistência específica, resistência à corrosão, baixa densidade e usinabilidade. As ligas de alumínio que respondem a tratamentos térmicos são chamadas de ligas endurecidas por precipitação ou ligas envelhecíveis, esses tratamentos incluem resfriamento rápido, precipitação e envelhecimento (natural ou artificial), já as ligas que não respondem a esses

IInstituto Federal de Educação, Ciência e Tecnologia de São Paulo - IFSP, Bragança Paulista, SP, Brasil. E-mail: torres@ifsp.edu.br; torres@fem.unicamp.br ${ }^{2}$ Departamento de Engenharia de Manufatura e Materiais, Faculdade de Engenharia Mecânica, Universidade Estadual de Campinas - UNICAMP, Campinas, SP, Brasil.

2176-1523 (C) 2019 Associação Brasileira de Metalurgia, Materiais e Mineração. Publicado pela ABM. Este é um artigo de acesso aberto distribuído sob os termos da licença Creative Commons CC BY-NC-ND (Attribution-NonCommercial-NoDerivs) - https:// creativecommons.org/licenses/by-nc-nd/4.0\%. 
tratamentos térmicos e são endurecíveis por trabalho a frio são chamadas de ligas endurecíveis por encruamento. Segundo classificação da American Society for Metals, as ligas da série $7 X X X$ contém como elemento principal o zinco, onde se destacam os subgrupos Al-Zn-Mg e Al-Zn-Mg-Cu que através do tratamento térmico (solubilização e envelhecimento) apresentam ganhos significativos de dureza. A principal aplicação destas ligas encontra-se na indústria aeronáutica, uma vez que atingem os níveis mais elevados de resistência mecânica entre as ligas de alumínio [I].

O tratamento térmico de solubilização tem como objetivo promover a dissolução das fases endurecedoras na matriz de alumínio; uma temperatura típica de solubilização para estas ligas seria em torno de $480^{\circ} \mathrm{C}$ evitando assim o superaquecimento e a fusão parcial do material. Na sequência ocorre um resfriamento rápido com o intuito de manter a solução sólida supersaturada e por fim um tratamento térmico de envelhecimento (endurecimento por precipitação) que pode ocorrer à temperatura ambiente ou superior, sendo chamado de natural ou artificial respectivamente [2].

As ligas de alumínio são as matérias-primas mais utilizadas nos processos que envolvem os materiais semissólidos, no entanto, as ligas alumínio-silício se destacam das outras ligas sendo utilizadas em $95 \%$ de todas as aplicações [3], sendo assim, este estudo visa contribuir para o desenvolvimento de novas ligas semissólidas, a saber, as ligas AA7004 e AA7075, devido às mesmas atingirem níveis elevados de resistência mecânica entre as ligas de alumínio [4] como também para o processo de tixoconformação, devido as suas inúmeras vantagens, tais como: menor consumo de energia, redução de etapas de produção, aumento da produtividade, aumento da vida útil de matrizes, obtenção de peças com excelente acabamento superficial e produção near-net-shape [5-7]. Portanto, este trabalho tem como objetivo efetuar a caracterização mecânica e metalúrgica das ligas tixoconformadas AA7004 e AA7075 antes e após o tratamento térmico de solubilização e envelhecimento, devido à importância tecnológica destas ligas.

\section{MATERIAIS E MÉTODOS}

A metodologia adotada para o desenvolvimento deste trabalho possui as seguintes etapas: caracterização das temperaturas de trabalho via ensaio de análise térmica de calorimetria diferencial de varredura - DSC, ensaios de tixoconformação, tratamento térmico de solubilização e envelhecimento natural, ensaios de dureza vickers e caracterização microestrutural.

\section{I Matéria-prima e Análise Térmica}

Neste trabalho foram utilizadas as ligas comerciais de alumínio AA7004 e AA7075 produzidas via lingotamento contínuo convencional pela empresa ALCOA Alumínio S.A, cujas composições químicas são: AA7004 (5,8\% Zn - I,5\% $\mathrm{Mg}-0,1 \% \mathrm{Cu}-0,4 \% \mathrm{Fe}-0,3 \% \mathrm{Si}-0,5 \% \mathrm{Mn}$ e Al balanço) e AA7075 (6,2\% Zn - 2,0\% Mg - I,7\% Cu - 0,5\% Fe - 0,4\% $\mathrm{Si}-0,1 \% \mathrm{Mn}$ e Al balanço). As temperaturas de trabalho das ligas correspondentes às frações líquidas de $40 \%$ e $55 \%$ foram determinadas por meio da técnica de análise térmica de calorimetria diferencial de varredura - DSC, modelo STA $409 \mathrm{C} \mathrm{NETZSCH}$ com uma taxa de aquecimento de $5^{\circ} \mathrm{C} / \mathrm{min}$ até a temperatura de $700^{\circ} \mathrm{C}$ e resfriamento até temperatura ambiente [8]. Esta técnica envolve a determinação do fluxo de calor absorvido ou liberado por uma amostra submetida a variações de temperatura, quando tal amostra sofre transformações de fases ocorre liberação ou absorção de energia, sendo detectado como fluxos de calor entre a amostra estudada e outra de referência [9]. Na Tabela I, tem-se o resumo das temperaturas encontradas [8], observa-se que a faixa entre as temperaturas solidus e liquidus $(\Delta \mathrm{T})$ apresenta-se inferior a $130^{\circ} \mathrm{C}$, demonstrando que ambas as ligas são propícias a serem utilizadas como matéria-prima no processo de tixoconformação segundo critérios estabelecidos por Liu et al. [10].

Com base nas composições químicas das ligas deste estudo, tem-se o diagrama de fases elaborado através do software Thermo-Calc ${ }^{\circledR}$ levando em conta a quantidade de alguns elementos químicos: para a liga AA7004 tem-se a quantidade de zinco e magnésio e para a liga AA7075 tem-se a quantidade de zinco, magnésio e cobre. $O$ processamento do material no estado semissólido ocorre entre a temperatura solidus e a temperatura liquidus, ou seja, onde há a coexistência da fase $\mathrm{Al}_{\mathrm{FCC}}$ juntamente com a fase líquida $\left(\mathrm{Al}_{\mathrm{FCC}}+\mathrm{L}\right)$, conforme visto em ambas as Figura la e Ib.

\subsection{Ensaios de Tixoconformação}

Os ensaios foram realizados em uma máquina universal de ensaios MTS, modelo 810 , com capacidade de carga de $100 \mathrm{kN}$, com um forno resistivo acoplado ao eixo da máquina, com capacidade de atingir uma temperatura máxima de trabalho de $1200^{\circ} \mathrm{C}$, com placas paralelas de Inconel 718 que possuíam área superior a área máxima ocupada pelas amostras deformadas, mantendo assim o volume deformado constante. As dimensões dos corpos de prova utilizados foram $15 \mathrm{~mm}$ de altura e $20 \mathrm{~mm}$ de diâmetro e com furo de I, $6 \mathrm{~mm}$ de diâmetro na metade de sua altura, submetidas a uma taxa de compressão constante de $10 \mathrm{~mm} / \mathrm{s}$ e tempo de execução de ensaio de I s. Após o

Tabela I. Temperaturas obtidas via ensaio de análise térmica de calorimetria diferencial de varredura

\begin{tabular}{cccccc}
\hline Ligas & $\mathbf{T}$ solidus $\left[{ }^{\circ} \mathbf{C}\right]$ & $\mathbf{T}$ liquidus $\left[{ }^{\circ} \mathbf{C}\right]$ & $\Delta \mathbf{T}\left[{ }^{\circ} \mathbf{C}\right]$ & $\mathbf{T}$ trabalho 40\%fl $\left[{ }^{\circ} \mathbf{C}\right]$ & $\mathbf{T}$ trabalho 55\%fl $\left[{ }^{\circ} \mathbf{C}\right]$ \\
\hline AA7004 & 603 & 662 & 59 & 642 & 647 \\
AA7075 & 548 & 636 & 88 & 607 & 614 \\
\hline
\end{tabular}



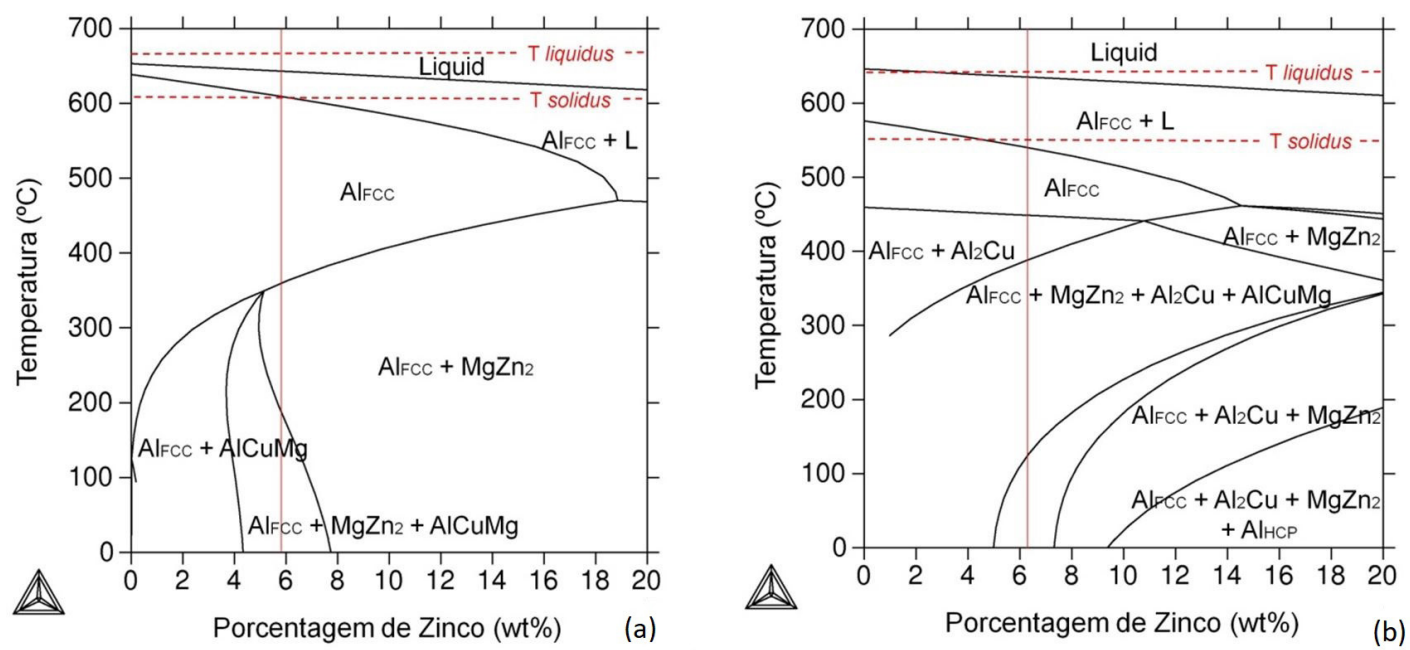

Figura I. Diagrama de fases das ligas AA7004 (a) e AA7075 (b) elaborado através do software Thermo-Calc ${ }^{\circledR}$ (banco de dados: TTAI5).
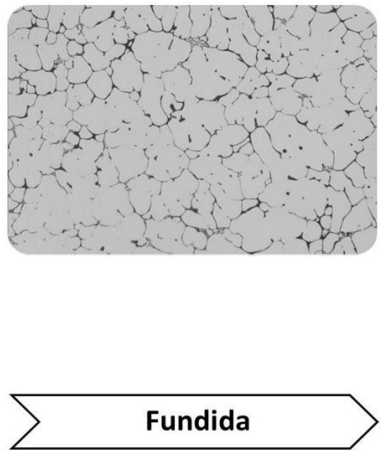
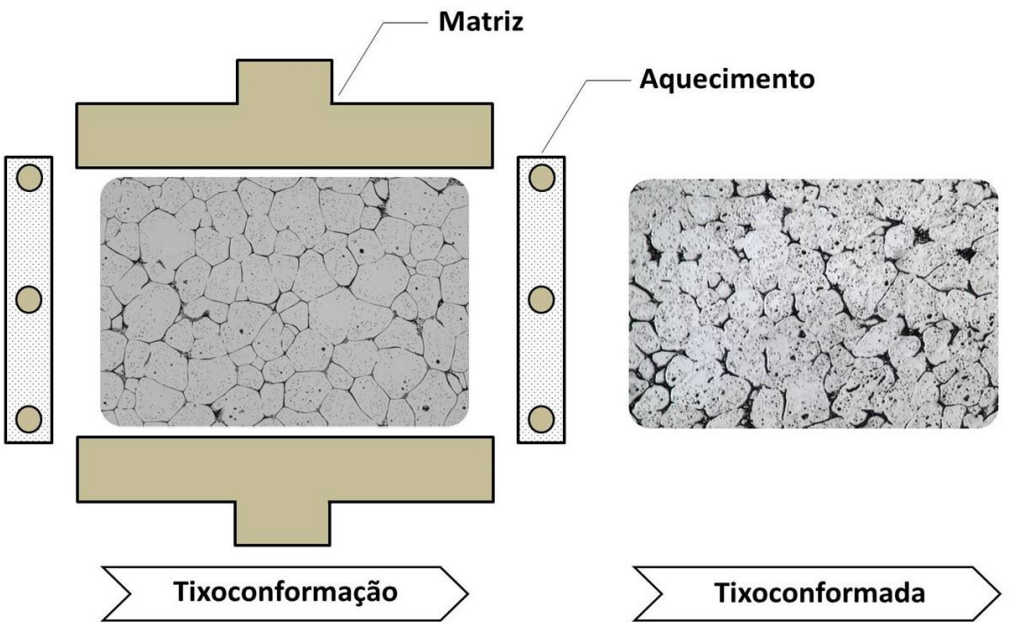

Figura 2. Sequência do ensaio de tixoconformação utilizando a liga AA7075 sob a condição de $40 \%$ fração líquida e tempo de globularização de 0 s.

sistema alcançar a condição requerida para cada situação, temperaturas relativas às frações líquidas de $40 \%$ e $55 \%$ e tempos de globularização de $0 \mathrm{~s}, 30 \mathrm{~s}$ e $90 \mathrm{~s}$ as amostras que foram inseridas juntamente com um termopar tipo $\mathrm{K}$ - Cromel Alumel fixado em seu interior foram comprimidas [ I I]. A sequência da realização do ensaio de tixoconformação, desde a condição fundida (com estrutura em formato de roseta), passando pelo tratamento térmico de globularização e tempo de espera (com estrutura globular) até a condição tixoconformada (estrutura globular deformada) pode ser visto na Figura 2; para evitar que as amostras se aderissem nas placas paralelas às mesmas foram pré-isoladas com cimento tipo QF 180; sendo realizadas três réplicas para cada condição analisada.

\subsection{Ensaios de Dureza Vickers}

O ensaio de dureza vickers consiste na aplicação de uma carga conhecida na superfície de um corpo de prova através de um penetrador piramidal de base quadrada, com ângulo entre as faces de $136^{\circ}$, fornecendo valores quantitativos da resistência à deformação superficial do material. Os ensaios foram realizados em um equipamento da marca Future-Tech, modelo FV-800, em amostras lixadas e polidas; foram realizadas 10 indentações em regiões aleatórias, porém sendo efetuadas no centro dos grãos, com carga de $5 \mathrm{kgf}$ aplicadas por um período de $15 \mathrm{~s}$ regido pela norma ASTM E92 [12].

\subsection{Tratamento Térmico de Solubilização e Envelhecimento}

O tratamento térmico de solubilização foi realizado em um forno resistivo e após o forno alcançar a temperatura desejada e sua consequente estabilização, as peças tixoconformadas foram inseridas em seu interior e reaquecidas até a temperatura relativa de seu tratamento, mantidas por tempo adequado e depois resfriadas em água, posteriormente passaram pelo processo de envelhecimento natural; a Figura 3a apresenta 

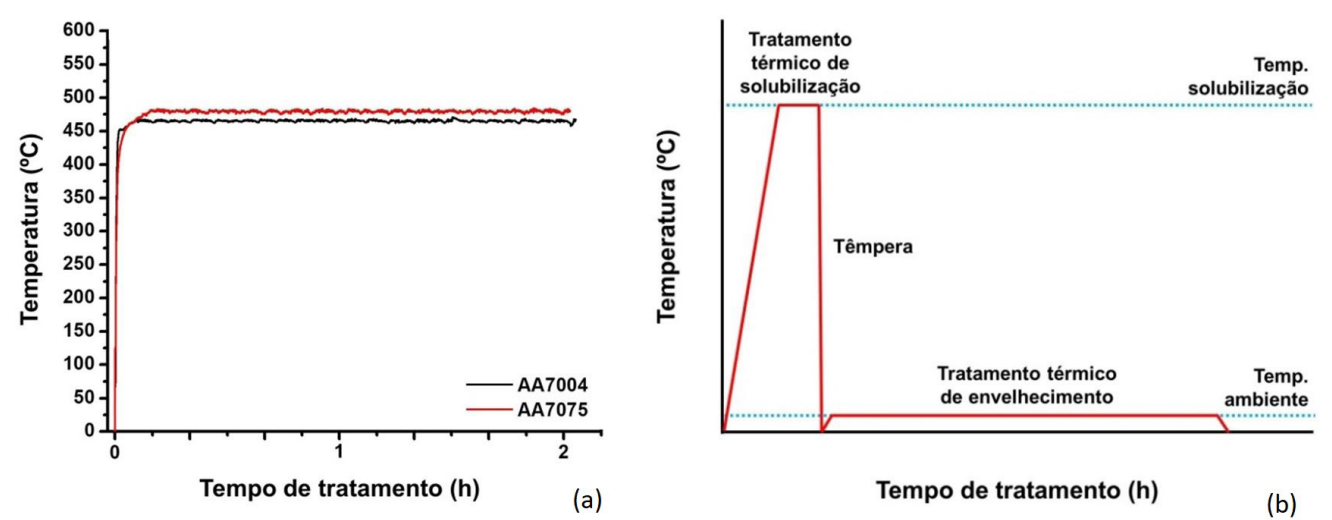

Figura 3. Tratamento térmico de solubilização realizado para ambas as ligas (a) e esquematização do ciclo completo do tratamento térmico de solubilização e envelhecimento natural (b).

- platô referente à temperatura de solubilização para ambas as ligas não havendo variações bruscas de temperatura e a Figura 3b apresenta a esquematização do ciclo completo do tratamento térmico realizado. Os parâmetros utilizados no tratamento térmico foram: temperatura do tratamento térmico de solubilização $\left(465^{\circ} \mathrm{C} \pm 2^{\circ} \mathrm{C}\right.$ para a liga AA7004 e $480^{\circ} \mathrm{C} \pm 2{ }^{\circ} \mathrm{C}$ para a liga AA7075) [I], tempo de tratamento (2 horas), têmpera em água $\left(24^{\circ} \mathrm{C} \pm 2^{\circ} \mathrm{C}\right)$ e tempo de envelhecimento natural ( 24 horas); o tempo de envelhecimento elevado ocorre devido aos processos de precipitação das fases que são muito mais lentos quando comparados com o envelhecimento artificial [I]. Sendo que, no tratamento térmico de solubilização ocorre a dissolução das fases $\mathrm{MgZn}_{2}$ e $\mathrm{Al}_{2} \mathrm{CuMg}$ na matriz de alumínio dando origem a uma solução sólida supersaturada enquanto que no envelhecimento pretende-se a obtenção controlada dos precipitados endurecedores, aumentando de forma significativa às propriedades mecânicas das ligas de alumínio.

\subsection{Caracterização Microestrutural}

As ligas foram caracterizadas microestruturalmente sob a condição fundida (como recebida), tixoconformada e após o tratamento térmico de solubilização e envelhecimento. As amostras foram lixadas em lixa d'água com granulometria de $220,320,400,600,800,1200$ e I 500 e polidas com pasta de diamante de $6 \mu \mathrm{m}$ e I $\mu \mathrm{m}$. As amostras foram atacadas com reagente Keller $\left(2,5 \mathrm{ml} \mathrm{HNO}_{3}, \mathrm{I}, 5 \mathrm{ml} \mathrm{HCl}, \mathrm{Iml} \mathrm{HF} \mathrm{e} 95 \mathrm{ml} \mathrm{H}_{2} \mathrm{O}\right)$ com a imersão total da amostra no reagente por um tempo de $10 \mathrm{~s}$ visando revelar sua microestrutura, após 0 ataque as amostras eram enxaguadas em água corrente por aproximadamente $30 \mathrm{~s}$ e secadas com auxílio de um soprador elétrico. Para a aquisição das imagens utilizou-se um microscópio óptico Leica DM ILM.

\section{RESULTADOS E DISCUSSÕES}

As micrografias da condição fundida (como recebida) de ambas as ligas são apresentadas na Figura 4a e 4d, nota-se uma microestrutura tipicamente em formato de roseta para a liga AA7075 e uma microestrutura mais grosseira, porém também em formato de roseta para a liga AA7004; considera-se a microestrutura com morfologia de roseta como sendo a intermediária entre a estrutura dendrítica e a estrutura globular [13]. Ao passar pelo tratamento térmico de globularização as ligas evoluem para uma morfologia globular mesmo para o tempo de tratamento térmico de $0 \mathrm{~s}$, como pode ser visto na Figura $4 \mathrm{~b}$ e $4 \mathrm{e}$, ocorrendo um pequeno aumento no tamanho dos glóbulos primários como também de seu grau de circularidade durante a manutenção do material à faixa semissólida para o tempo de 90 s de tratamento. A evolução para a microestrutura globular deve-se aos fenômenos de ostwald ripening e coalescência, ambos dependentes do tempo de permanência da liga à temperatura de tratamento térmico $[14,15]$. A análise microestrutural das peças tixoconformadas deram-se na área central da peça, ou seja, onde o material sofre alta deformação até os estágios finais da conformação, sendo constituídos de glóbulos primários deformados, vistos na Figura 4c e 4f, pois no estado semissólido o líquido guiado por gradientes de pressão é rejeitado para as extremidades da peça sendo que no centro da peça praticamente não há presença de líquido, fato observado em ambas as micrografias tixoconformadas, onde se tem uma menor quantidade de contorno de grãos (eutético). A Figura 4 apresenta apenas a condição de $40 \%$ de fração líquida e tempo de globularização de $0 \mathrm{~s}$, pois todos os fatos reportados para esta condição foram os mesmos encontrados paras as demais condições analisadas.

A Figura 5 apresenta os resultados obtidos nos ensaios de dureza vickers sob diferentes condições de frações líquidas e tempos de tratamento de globularização de ambas as ligas. Nota-se que os maiores valores ocorreram com a menor fração líquida, o que facilita os fenômenos de coalescência devido ao contato entre as partículas sólidas, propiciando uma microestrutura globular deformada. A condição fundida apresenta valores muito inferiores quando comparada com a condição tixoconformada, ou seja, $53 \mathrm{HV}$ para a liga AA7004 e 68 HV para a liga AA7075 [16], uma vez que a condição fundida possui microestrutura em formato de roseta, ou 

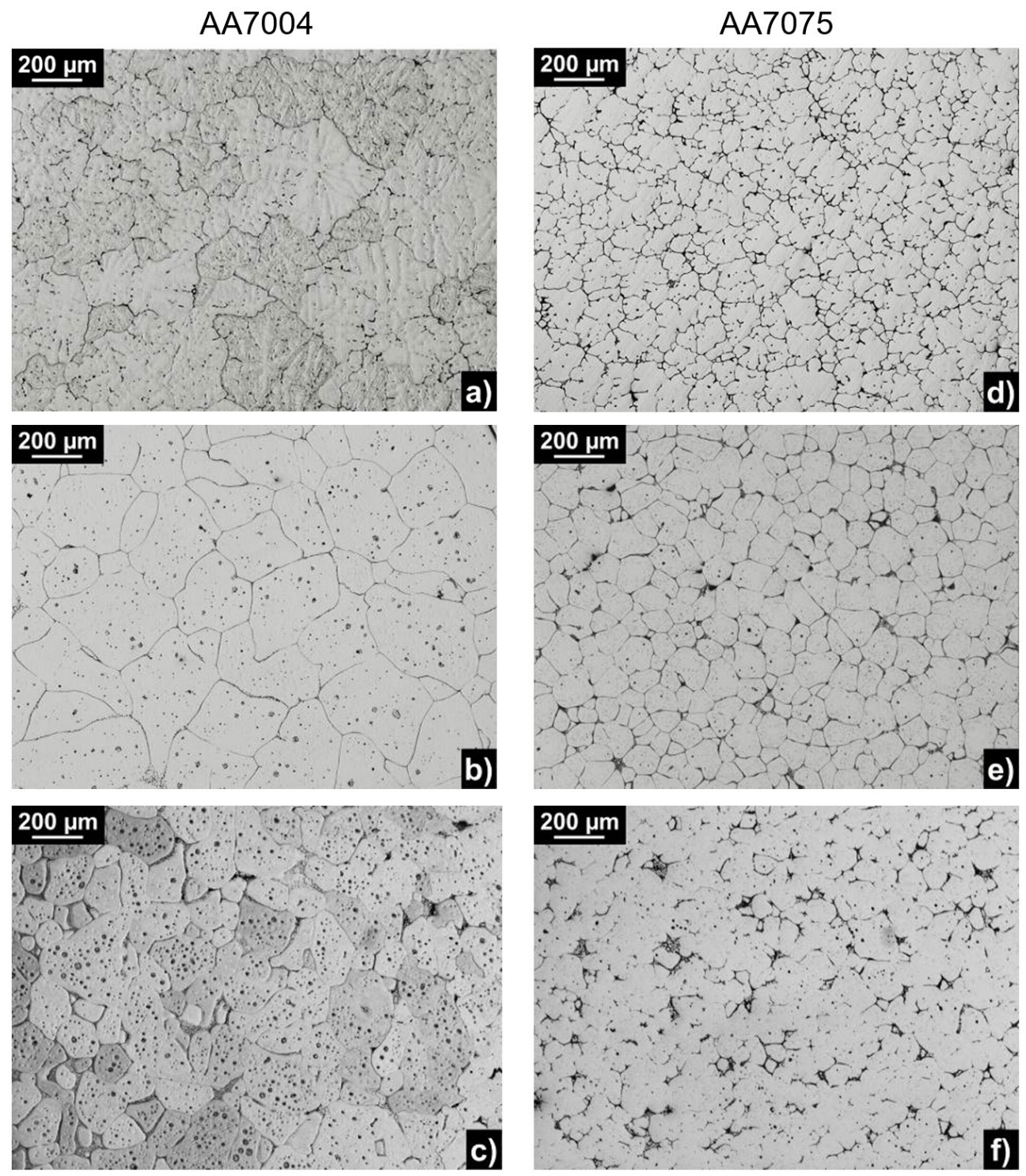

Figura 4. Micrografias das ligas fundidas (a, d), tratadas termicamente para $40 \%$ de fração líquida e tempo de globularização de 0 s (b, e) e as ligas tixoconformadas, solubilizadas e envelhecidas (c, f).

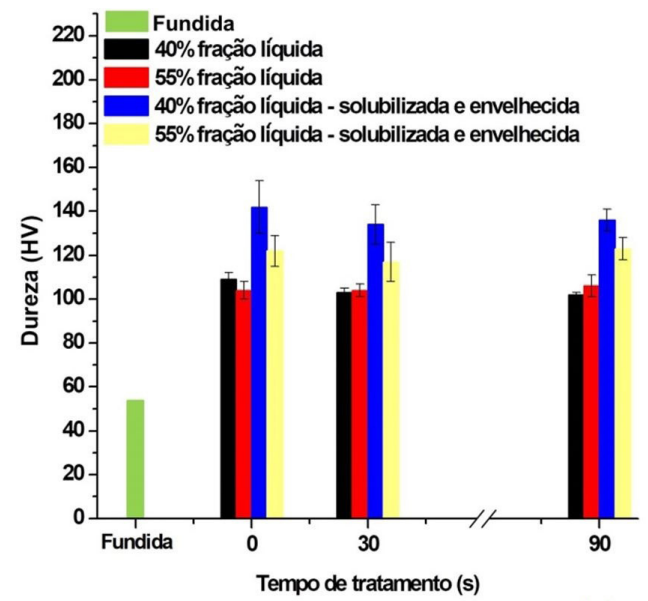

(a)

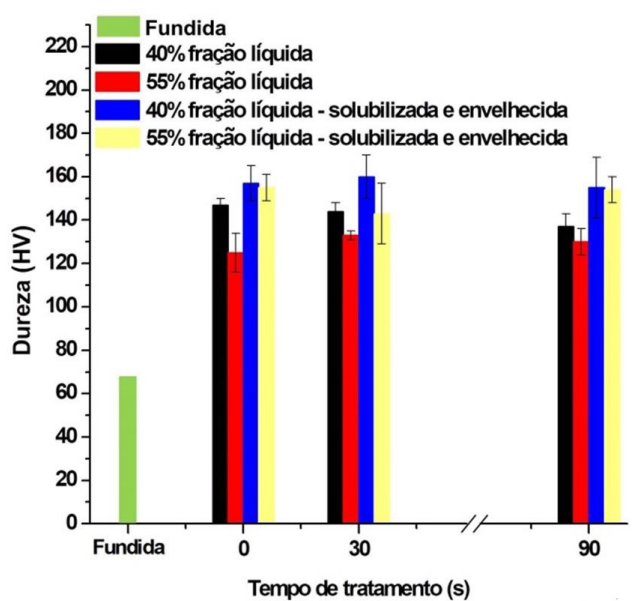

(b)

Figura 5. Dureza vickers das ligas AA7004 (a) e AA7075 (b) tratadas termicamente para $40 \%$ e $55 \%$ de fração líquida e tempos de globularização de 0 s, 30 s e 90 s, sob todas as condições. 
seja, uma microestrutra intermediária à condição globular. Um fator importante no aumento considerável nos valores de dureza da condição tixoconformada decorre à alta deformação sofrida na compactação do material durante o processo de tixoconformação; assim, tem-se para a liga AA7004 um aumento médio de dureza em torno de $75 \mathrm{HV}$ e para a liga AA7075 um aumento ainda maior em torno de $85 \mathrm{HV}$. Após o tratamento térmico de solubilização e envelhecimento natural nas peças tixoconformadas, têm-se resultados ainda mais satisfatórios, a liga AA7004 apresenta valores médios de dureza em comparação com a liga apenas tixoconformada em torno de $25 \mathrm{HV}$ e a liga AA7075 valores médios de dureza em torno de $20 \mathrm{HV}$, ou seja, o tratamento térmico propiciou o aumento na dureza dos materiais devido à dissolução de algumas fases na matriz de alumínio como também devido à obtenção dos precipitados endurecedores. Os tempos de tratamento de globularização ( 0 s, 30 s e 90 s) apresentaram homogeneidade não havendo grandes variações nos valores de dureza, com isso pode-se concluir a influência da fração líquida (temperatura de trabalho) no aumento da dureza de ambas as ligas. Vieira [17] analisando a liga AA7075 com 55\% de fração líquida obteve valores de dureza média de $94 \mathrm{HV}$, ou seja, valores inferiores das ligas apenas tixoconformadas deste trabalho, mostrando que a utilização do processo de tixoconformação propicia um aumento destes valores. Souza [18] analisando a liga AA7075 em diferentes condições: solubilizada, superenvelhecida e superenvelhicida/laminada a frio/recozida obteve baixos valores, a saber, $94 \mathrm{HV}$ para a liga solubilizada, $82 \mathrm{HV}$ para a liga superenvelhecida e para a condição superenvelhicida/laminada a frio/recozida os valores foram ainda menores de aproximadamente $80 \mathrm{HV}$.

\section{CONCLUSÕES}

Tem-se as seguintes conclusões para as ligas AA7004 e AA7075 tixoconformadas:

- Possuem estabilidade no estado semissólido, apresentando morfologia globular para as diferentes frações líquidas como também para os diferentes tempos de tratamento de globularização, mostrando que os fenômenos de ostwald ripening e coalescência, ambos dependentes do tempo de permanência da liga à temperatura semissólida agiram simultaneamente;

- Apresentam valores de dureza vickers muito superiores quando comparados com a condição fundida, a saber, para a liga AA7004 um aumento de $75 \mathrm{HV}$ e para a liga AA7075 um aumento de $85 \mathrm{HV}$, devido às mesmas terem sido tixoconformadas. Quando submetidas ao tratamento térmico de solubilização e envelhecimento natural, houve outro aumento nos valores de dureza, melhorando de forma significativa as propriedades mecânicas das ligas.

\section{Agradecimentos}

Os autores agradecem a empresa ALCOA Alumínio S.A. pela doação da matéria-prima e a CAPES (Coordenação de Aperfeiçoamento de Pessoal de Nível Superior) por seu apoio financeiro. Gostaríamos também de agradecer ao Departamento de Engenharia de Manufatura e Materiais da Faculdade de Engenharia Mecânica da Universidade Estadual de Campinas - DEMM/FEM/UNICAMP e ao Instituto Federal de Educação, Ciência e Tecnologia de São Paulo - IFSP, campus Bragança Paulista.

\section{REFERÊNCIAS}

I American Society for Metals. Aluminum: properties and physical metallurgy. Ohio: ASM Metals Park; 1990. Metals handbook.

2 Soares MRN. Efeitos dos ciclos de envelhecimento no comportamento de ligas de alumínio [dissertação]. Aveiro: Universidade de Aveiro; 2009.

3 Chiarmetta G. Why thixo? In: Proceedings of the VI International Conference on the Semi-solid Processing of Alloys and Composites; 2000 September 27-29; Turin, Itália. Turin: Edimet; 2000. p. I5-2I.

4 Infomet [página da internet]. Rio de Janeiro: INFOMET; 2018 [acesso em 10 jun. 20I8]. Disponível em: http://www. infomet.com.br/site/metais-e-ligas-conteudo-ler.php?codAssunto $=55$

5 Brown SB, Flemings MC. Net shape forming via semi-solid processing. Advanced Materials \& Processes. 1993; 1:36-40.

6 Kapranos P, Ward PJ, Atkinson HV. Near net shaping by semi-solid metal processing. Materials \& Design. 2000;21:387-394.

7 Atkinson HV. Modeling the semi-solid processing of metallic alloys. Progress in Materials Science. 2005;50:34I-4I2.

8 Zoqui EJ, Torres LV. Evaluation of the thixoformability of AA7004 and AA7075 alloys. Materials Research. 2010;13:305-318. 
9 Opini VC. Preparação, processamento e caracterização de ligas de titânio com alta resistência mecânica baseadas na liga Ti-5553 [dissertação]. Campinas: Universidade Estadual de Campinas; 2012.

10 Liu D, Atkinson HV, Jones $\mathrm{H}$. Thermodynamic prediction of thixoformability in alloys based on the Al-Si-Cu and Al-Si-Cu-Mg systems. Acta Materialia. 2005;53(14):3807-38I9.

I I Torres LV. Avaliação da tixoconformabilidade das ligas AA7075 e AA7004 [dissertação]. Campinas: Universidade Estadual de Campinas; 2009.

12 American Society for Testing and Materials. ASTM E92: Standard test methods for vickers and knoop hardness of metallic materials. ASTM: West Conshohocken; 2016.

13 Flemings MC. Behavior of metal alloys in the semi-solid state. Metallurgical Transactions. A, Physical Metallurgy and Materials Science. 1991;22(5):957-981.

14 Genda G, Yuyon C, Geing A. Mechanism of coarsening of dendrite during solidification, In: Proceedings of the Solidification Processing Conference; 1987 September; Sheffield, London. USA: N. American Publications Center; 1987. p. 416-4I9.

15 Kattamis TZ, Coughin JL, Flemings MC. Influence of coarsening on dendritic arm spacing of Al-Cu alloys. Transactions of the Metallurgical Society of AIME. 1967;239:I504-I5II.

16 Matweb [página da internet]. Blacksburg: MATWEB; 2018 [acesso em 10 nov. 2018]. Disponível em: http://www. matweb.com/search/CompositionSearch.aspx

17 Vieira MCB. Caracterização da viscosidade aparente no estado semi-sólido e das propriedades mecânicas da liga AA7075 [tese]. Porto Alegre: Universidade Federal do Rio Grande do Sul; 2013.

18 Souza SH. Cinética de amolecimento da liga de alumínio AA7075 durante recozimento após laminação a frio [dissertação]. São Paulo: Escola Politécnica, Universidade de São Paulo; 2018.

Recebido em: 15 Nov. 2018

Aceito em: 2 Abr. 2019 